\title{
Immunotherapy Prospects for Painful Small-fiber Sensory Neuropathies and Ganglionopathies
}

\author{
Anne Louise Oaklander ${ }^{1}$
}

Published online: 2 November 2015

(C) The American Society for Experimental NeuroTherapeutics, Inc. 2015

\begin{abstract}
The best-known peripheral neuropathies are those affecting the large, myelinated motor and sensory fibers. These have well-established immunological causes and therapies. Far less is known about the somatic and autonomic "small fibers"; the unmyelinated C-fibers, thinly myelinated A-deltas, and postganglionic sympathetics. The small fibers sense pain and itch, innervate internal organs and tissues, and modulate the inflammatory and immune responses. Symptoms of small-fiber neuropathy include chronic pain and itch, sensory impairment, edema, and skin color, temperature, and sweating changes. Small-fiber polyneuropathy (SFPN) also causes cardiovascular, gastrointestinal, and urological symptoms, the neurologic origin of which often remains unrecognized. Routine electrodiagnostic study does not detect SFPN, so skin biopsies immunolabeled to reveal axons are recommended for diagnostic confirmation. Preliminary evidence suggests that dysimmunity causes some cases of small-fiber neuropathy. Several autoimmune diseases, including Sjögren and celiac, are associated with painful small-fiber ganglionopathy and distal axonopathy, and some patients with "idiopathic" SFPN have evidence of organ-specific dysimmunity, including serological markers. Dysimmune SFPN first came into focus in children and teenagers as they lack other risk factors, for example diabetes or toxic exposures. In them, the rudimentary evidence suggests humoral rather than cellular mechanisms and complement consumption. Preliminary evidence supports efficacy of corticosteroids
\end{abstract}

Anne Louise Oaklander

aloaklander@mgh.harvard.edu

1 Department of Neurology and Department of Pathology (Neuropathology) Massachusetts General Hospital, Harvard Medical School, Boston, MA 02114, USA and immunoglobulins in carefully selected children and adult patients. This paper reviews the evidence of immune causality and the limited data regarding immunotherapy for small-fiberpredominant ganglionitis, regional neuropathy (complex regional pain syndrome), and distal SFPN. These demonstrate the need to develop case definitions and outcome metrics to improve diagnosis, enable prospective trials, and dissect the mechanisms of small-fiber neuropathy.

Keywords Ganglionitis - Complex regional pain syndrome · Polyneuropathy $\cdot \mathrm{C}$-fiber $\cdot$ Neuropathic pain $\cdot$ Small-fiber neuropathy

\section{Introduction}

Peripheral neurons used to be neatly categorized into motor, sensory, or autonomic. Sensory neurons were divided into large myelinated low-threshold receptors versus smalldiameter high-threshold receptors for painful stimuli (nociception). Then molecular study blurred the distinction between nociceptive and autonomic axons. The culprit is the small-diameter unmyelinated C-fiber, which does far more than sense pain. This evolutionarily primitive neuron functions bidirectionally_ its distal axon terminal not only transduces and transmits pain, but it also releases electrical and paracrine chemical signals that maintain tissue homeostasis. C-fiber trophic signals are critical for the integrity of skin and bone for instance $[1,2]$. Other $\mathrm{C}$-fiber paracrine secretions modulate tissue responses to injury or threat. In "neurogenic inflammation" C-fibers release calcitonin-gene related peptide (CGRP) and substance $\mathrm{P}$ neuropeptides from their distal axon terminals. These trigger vasodilation, plasma protein extravasation, tissue edema, hemoconcentration, and leukocyte diapedesis [3]. C-fibers also modulate immune responses, with mast cells 
in close physical and chemical contact [4]. In turn, C-fiber signaling is modified by inflammatory signals, including serotonin, histamine, glutamate, adenosine triphosphate, adenosine, substance P, calcitonin-gene related peptide, bradykinin, eicosanoids prostaglandins, thromboxanes, leukotrienes, endocannabinoids, nerve growth factor, inflammatory cytokines, and protons. These bind to C-fiber surface receptors, including $\mathrm{G}$ protein-coupled receptors, transient receptor potential channels, acid-sensitive ion channels, 2-pore potassium channels, and receptor tyrosine kinases, as reviewed in Basbaum et al. [5]. These allow injury and inflammation to trigger and sensitize pain signaling. Crosstalk between nociceptive and immune cells contributes to host defense and immune-mediated diseases [6], and there is preliminary evidence that disturbed immunity underlies some cases of painful small-fiber neuropathy (SFN). This suggests the novel possibility that immunotherapy might be a more effective treatment for pain (and other symptoms) than pain relievers in such patients. Given the lackluster long-term efficacy, potentially disastrous complications of chronic opioid therapy, and its lack of disease-modifying activity, this hypothesis deserves further consideration.

\section{Immune-mediated Painful Sensory Ganglionitis/Neuronitis}

Anatomy attests to the importance of small-fiber neurons in protecting us against harm. Transmitting pain signals is merely one part of their larger role. During fetal development, these neural crest derivatives migrate from the central nervous system, forsaking the shelter of the blood-brain barrier to function as sentinels, positioned to detect and respond rapidly to environmental threats. To that end, the capillaries in sensory and somatic ganglia are fenestrated. This exposes the neuron cell bodies to blood-borne signals but leaves them vulnerable to toxins, infections (e.g., HIV, varicella zoster virus), and immune attack $[7,8]$. The symptoms of ganglionopathy depend on the type of neuron targeted. Damage to large myelinated sensory neurons reduces touch, vibration, and proprioception, causing ataxia, athetoid limb movements, and difficulty moving, despite preserved strength [9]. Less attention has been paid to the symptoms caused by attacking smallfiber neurons; distal chronic neuropathic pain, itch, and sometimes swelling and redness. Sensory ganglionopathies differ from distal sensory axonopathies by their patchy, asymmetric, and often proximal signs and symptoms, and they sometimes have more rapid onset. Ganglionitis, particularly from Sjögren syndrome (SS), should be considered in patients with sudden proximal or patchy unexplained neuropathic pain (Fig. 1). Rare patients may require ganglion biopsy to confirm the presence of ganglionitis and inform treatment decisions. Characteristic pathological findings include neuronal loss,

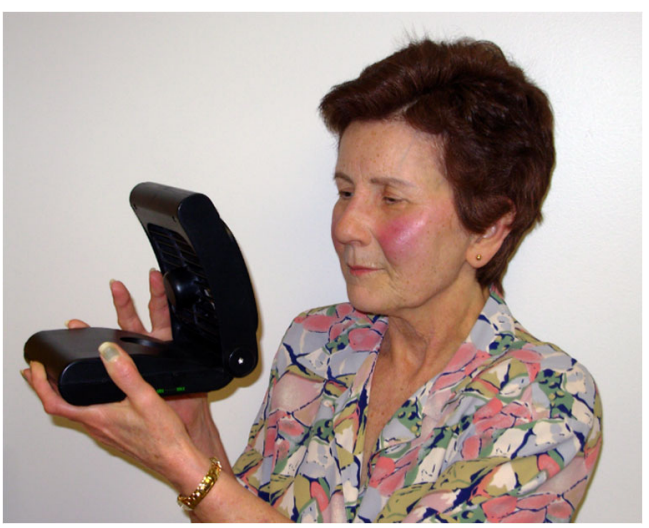

Fig. 1 A woman with unexplained chronic pain and redness in both cheeks that she cooled with a fan (facial erythromelalgia). This was later recognized as seronegative Sjögren syndrome-associated trigeminal ganglionopathy. She later developed pain in her feet and hands; distal leg skin biopsy confirmed small-fiber distal axonopathy. Immunotherapies included long-term corticosteroids, quinacrine (which discolored her nails), and intravenous immunoglobulin. Isolated trigeminal neuropathy was reported in $7 \%$ of a series of 30 patients with Sjögren's syndrome-associated neuropathy [15]; many cases are seronegative

Nageotte nodules, and mononuclear infiltrates [10]. In paraneoplastic ganglionitis, immunohistochemical analysis has shown intraneural IgG deposits without complement deposition [5]. Less invasive tests for ganglionitis include highresolution spine magnetic resonance imaging, which sometimes reveals hyperintense T2-weighted lesions or volume reduction in ganglia or in the dorsal columns. Cerebrospinal fluid can have high protein, cells, or oligoclonal $\operatorname{IgG}$ bands. Electrodiagnostic studies can disclose large-fiber involvement such as reduced or absent sensory nerve action potentials [7], and skin biopsies can reveal proximal or nonlength-dependent loss of small-fiber innervation [11]. These tests can objectively confirm the diagnosis and prompt consideration of immunotherapy, but negative results do not exclude the diagnosis. Among autoimmune ganglionitides, SS is best known, but rheumatoid arthritis, systemic lupus erythematosus, and autoimmune hepatitis are also implicated [8]. Celiac, another autoimmune condition associated with ganglionitis, is discussed in the section on polyneuropathy as most of the studies did not distinguish between ganglionopathy and distal axonopathy $[12,13]$.

In small-fiber predominant Sjögren syndrome, patients have relative sparing of large-fiber modalities and instead have symptoms of SFN (Fig. 1). In one study, pure SS small-fiber ganglionitis was defined by the presence of painful sensory symptoms with normal nerve conduction studies and abnormal neurophysiologic tests for small nerve fibers or a low epidermal fiber density within skin biopsy (Fig. 2) [14]. In a French study using this definition, $93 \%$ of patients were female and they were far less likely to have serological markers of Sjögren's [14]. In a hospital-based 


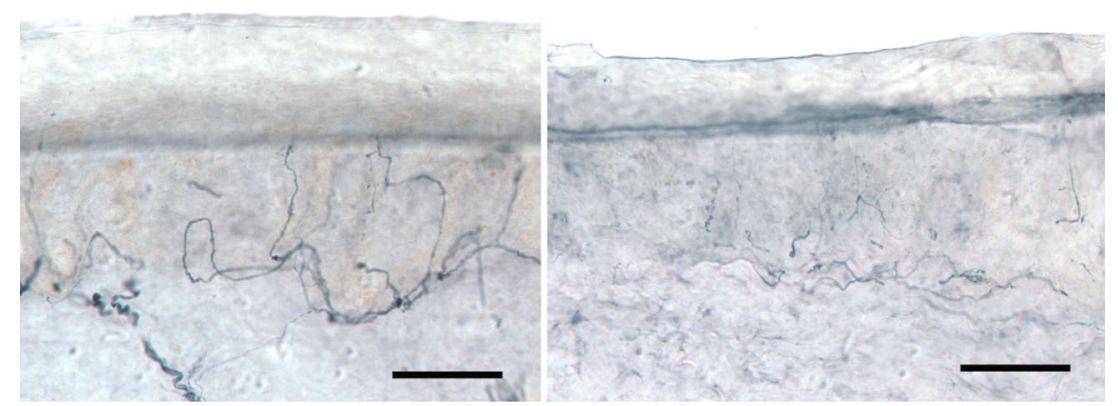

Fig. 2 Loss of PGP 9.5 immunolabeled nerve fibers in vertical sections from distal leg skin biopsy is the chief test used to objectively confirm small-fiber polyneuropathy. (A) Biopsy from a healthy 19-year-old white male control contains abundant innervation ( 675 fibers $/ \mathrm{mm}^{2}$ skin surface area). (B) Biopsy from a 19-year-old white male with small-fiber

survey of 120 patients with SS, $25 \%$ had definite neuropathy, with pure small-fiber attribution most common [15]. Not all patients with SS neuropathy report dry eyes or mouth, and in a series of 20 patients, only $55 \%$ were seropositive for SS-A/SS-B antibodies [16, 17]. In seronegative patients with high clinical suspicion, biopsy of minor (lip) salivary glands and/or testing for anti-Ro52/TRIM21 antibodies should be considered [18]. The few pathological studies of SS-affected ganglia document infiltration of mononuclear and predominantly $\mathrm{T}$ cells, associated with cellular degeneration, lack of axonal sprouts, and no evidence of frank vasculitis. [10]

There is no consensus on how best to treat Sjögren syndrome (SS). Some patients receive no immunotherapy, and among those treated, there is no standard regime [16, 17]. Common immunotherapies include hydroxychloroquine low-dose glucocorticoids ( $<20 \mathrm{mg} /$ day), and high-dose glucocorticoids $(>20 \mathrm{mg} /$ day). Second-tier options include rituximab (RTX) and intravenous immunoglobulins (IVIg) [19]. Their relative benefit for neuropathy is unknown, as most randomized clinical trials (RCT) for SS did not include neurological end points. A recent RCT reported no benefit of hydroxychloroquine $400 \mathrm{mg} / \mathrm{d}$ or RTX (1 g given twice) for pain or other outcomes [20, 21]. Small case series support the use of IVIg for ataxic (large-fiber) sensory ganglionitis [22]. The best evidence comes from a French multicenter study documenting efficacy of IVIg $2 \mathrm{~g} / \mathrm{kg}$ for SS neuropathy, including nonataxic (small-fiber) ganglionitis [23].

Another immunological cause of ganglionitis is paraneoplastic autoimmunity associated with antibodies to $\mathrm{Hu}$ or to the collapsin response mediator protein-5 (CRMP5) $[24,25]$. Small-cell lung tumors are particular triggers because of their neuroendocrine origin. The key therapy is tumor ablation, which removes antigenic drivers and effects cure, but while searching for the tumor, or if no tumor is found, immunotherapy is mandatory. Sensory ganglionitis has not been a primary outcome for clinical trials; thus, agents with documented efficacy against other neurological symptoms are used polyneuropathy demonstrates reduced epidermal $\left(155\right.$ fibers $\left./ \mathrm{mm}^{2}\right)$ and dermal nerve fibers. The fiber density at the $1.3 \mathrm{rd}$ centile of laboratory norms pathologically confirms his diagnosis. Bars represent $50 \mu \mathrm{m}$. Reproduced with permission from [74]

[26, 27], usually beginning with corticosteroids and IVIg [28]. One tumorless patient with anti-Hu sensory neuronopathy and gastric dysmotility achieved sustained improvement for 2 years after RTX treatment [29]. Assessing the outcomes of immunotherapy treatment for ganglionitis is complicated by the fact that stopping the immune attack may not improve symptoms caused by earlier death of neurons. For instance, postherpetic neuralgia can persist long after the shingles ganglionitis resolves [30]. However, usually it gradually improves and often resolves, presumably reflecting sprouting of axons from surviving cell bodies into deafferented targets.

\section{Complex Regional Pain Syndrome}

The pathogenesis of this historically rich post-traumatic neuropathic pain syndrome is still incompletely understood. One core feature is painful mono- or oligo- post-traumatic neuropathy in 1 extremity [31]. Ninety percent of cases are triggered by trauma (sprains/strains, fractures, iatrogenic), and some of the nontraumatic cases follow known or suspected focal nerve lesions, for example infarction, entrapment, and shingles [32, 33]. Complex regional pain syndrome (CRPS) is definitionally distinguished from post-traumatic neuralgia by additional visible signs, specifically "edema, changes in skin blood flow, or abnormal sudomotor activity in the region of the pain" [34] (Fig. 2). Many symptoms reflect microvascular dysfunction in a limb, and there is emerging evidence of a role for autoimmunity.

American neurologist S. Weir Mitchell first characterized CRPS in American Civil War soldiers with major penetrating nerve injuries as "causalgia" from the Greek causos (heat) and algia (pain) [35, 36], and in 1900 Sudeck first identified its effects on bones and joints [37-39]. In 1946, Evans described similar distal limb symptoms in patients without known nerve injuries that he called "reflex sympathetic dystrophy" [40]. Other historical names include "algodystrophy", "shoulderhand syndrome", and "Sudeck's atrophy" [37]. In 1994 the International Association for the Study of Pain created the 
"complex regional pain syndrome" terminology with the CRPS-I subtype replacing reflex sympathetic dystrophy for patients without documented nerve injuries, and CRPS-II replacing causalgia for patients with localized nerve injuries [34]. As the means by which nerve injuries should be diagnosed was never specified, and partial or distal nerve injuries often remain undiagnosed, for instance those caused by phlebotomy [41], the distinction between CRPS-I and CRPS-II is dubious (see Table 1 for the current International Association for the Study of Pain diagnostic criteria). Early concerns that CRPS was caused by psychopathology were resolved by pathological demonstration of somatotopic dysfunction and degeneration of small unmyelinated axons (Fig. 3) [42-44], and epidemiological studies finding no association with psychopathology [45, 46]. As CRPS resolves, which it usually does even without treatment [47], the edema, temperature changes, hyperhidrosis, and abnormal hair growth usually

Table 1 The 2003 Budapest diagnostic criteria for complex regional pain syndrome (CRPS), endorsed by the International Association for the Study of Pain in 2012. Distinctions between CRPS-I and CRPS-II are retained and a third subtype, CRPS-NOS (not otherwise specified) is recommended. Reproduced with permission from [96]

\section{General definition}

CRPS describes an array of painful conditions characterized by continuing (spontaneous and/or evoked) regional pain seemingly disproportionate in time or degree to the usual course of any known trauma or other lesion. The pain is regional (not in a specific nerve territory or dermatome) and usually has a distal predominance of abnormal sensory, motor, sudomotor, vasomotor, and/or trophic findings. The syndrome shows variable progression over time.

To make the clinical diagnosis the following criteria must be met:

1. Continuing pain, which is disproportionate to any inciting event

2. Must report at least 1 symptom in 3 of the 4 following categories: Sensory: reports of hyperesthesia and/or allodynia

Vasomotor: reports of temperature asymmetry and/or skin color changes and/or skin color asymmetry

Sudomotor/edema: reports of edema and/or sweating changes and/or sweating asymmetry

Motor/trophic: reports of decreased range of motion and/or motor dysfunction (weakness, tremor, dystonia) and/or trophic changes (hair, nail, skin)

3. Must display at least 1 sign at time of evaluation in 2 or more of the following categories:

Sensory: evidence of hyperalgesia (to pinprick) and/or allodynia (to light touch and/or temperature sensation and/or deep somatic pressure and/or joint movement)

Vasomotor: evidence of temperature asymmetry $\left(>1^{\circ} \mathrm{C}\right)$ and/or skin color changes and/or asymmetry

Sudomotor/edema: evidence of edema and/or sweating changes and/ or sweating asymmetry

Motor/trophic: evidence of decreased range of motion and/or motor dysfunction (weakness, tremor, dystonia) and/or trophic changes (hair, nail, skin)

4. There is no other diagnosis that better explains the signs and symptoms

For research purposes: must report at least 1 symptom in all 4 symptom categories and at least 1 sign (observed at evaluation) in 2 or more sign categories resolve before the pain $[48,49]$, so late-stage patients often no longer meet diagnostic criteria for CRPS but only for posttraumatic neuralgia, demonstrating that these are on a continuum (Fig. 4).

Histological study shows no inflammatory cellular infiltrates in CRPS-affected tissues [50]. The inflammation is chiefly neurogenic and microvascular [51]. The distal limb is vulnerable because it lacks collateral circulation and blood must overcome gravity to exit. Leaky microvessels cause several of the characteristic signs of small-fiber damage: edema, sometimes including blisters, and abnormal color and temperature (Fig. 2) [52]. The intravascular hemoconcentration associated with microvascular leaking promotes diapedesis of inflammatory cells into the limb. CRPS microvasculopathy has been attributed to malfunction and degeneration of the smallibers innervating affected microvessels distal to the causal injury (Fig. 3) [43]. Denervation of the arteriovenous shunts within CRPS-affected limbs permits blood to flow directly from the arterioles to the venules, bypassing the capillary beds and leaving the skin engorged with blood, while the deep tissues starve for oxygen and nutrients [43]. This hypoxia triggers further inflammation and pain [53, 54].

The literature variably documents elevated levels of proinflammatory cytokines and other inflammatory markers in blood and tissues from patients with CRPS [55]; however, these abate over time [56]. Few studies included other chronic pain controls so the findings may not be CRPS-specific [57, 58]. Limited evidence suggests that CRPS symptoms are not improved by treatment with nonsteroidal anti-inflammatory medications [59]. Corticosteroids are effective for acute CRPS but not for most chronic cases [59]. Adalimumab, a recombinant human monoclonal antibody that blocks tumor necrosis factor (TNF- $\alpha$ ) binding was tested in an uncontrolled study of 10 patients with CRPS-I, with 6 responders [60]. Case reports and a study of 42 patients reported efficacy of thalidomide, a tumor necrosis factor- $\alpha$ inhibitor [61], but a phase II, blinded RCT of lenalidomide, a safer thalidomide derivative, in 184 patients with CRPS-I did not find efficacy [62]. Perhaps it would have been more effective if given earlier in the course.

Recent evidence implicates autoimmunity in CRPS [63]. Sera from patients with CRPS has been reported to harbor autoantibodies targeting autonomic neurons [64, 65], and IgG extracted from these sera reproduce some CRPS features in mice [66]. These early passive transfer experiments suggest roles for B cells and IgM [67], and IgG autoantibodies [66]. Additionally, there is strong pathological and physiological evidence that focal injury to nerves sustained during surgery (which breaches the blood-nerve barrier) sometimes triggers autoimmune neuropathy [68]. Although CRPS was not referenced, surgeries are a common trigger for CRPS, and it is logical to assume that nonsurgical traumas could also trigger antinerve autoimmunity. Also, one group has reported modest efficacy of immunomodulatory therapies for established 


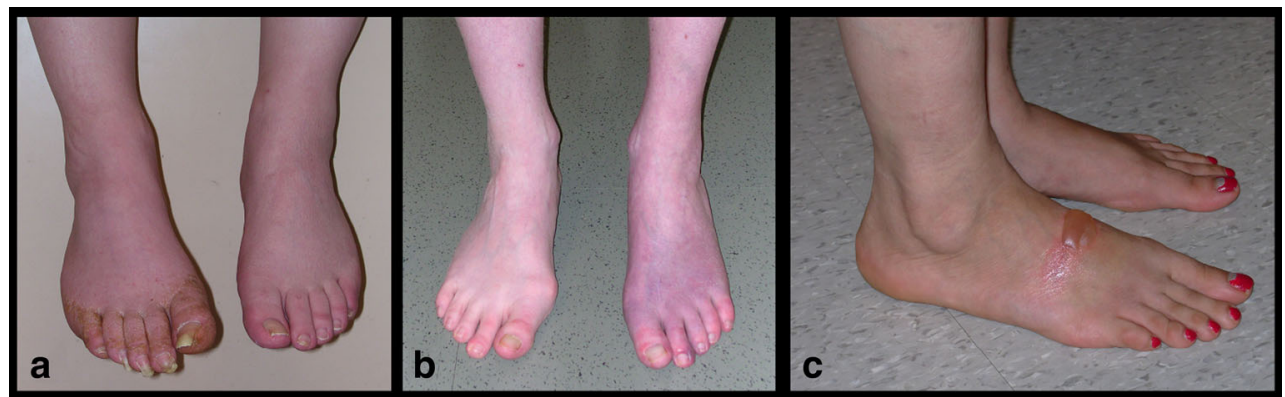

Fig. 3 Complex regional pain syndrome is a "pain-plus" syndrome affecting $1 \mathrm{limb}$, usually distal to a trauma. Focal nerve damage (mononeuropathy) contributes. (A) A laborer 4 years after right-foot crush injury left severe pain in the great toe that spread to the entire foot, which he guarded from contact, even from bathwater. Poor hygiene, mild edema, color change, and toe dystonia are visible. The history and a Tinel's sign at his right fibular head suggested peroneal nerve injury, but he was afraid to undertake electrodiagnostic study. (B)
A woman with chronic pain, edema, and vasodysregulation in the left foot and lower leg following bunionectomy years prior. There was also heterotopic ossification of the left tibia. (C) An otherwise healthy woman several years after healed second-degree burn from a hot coffee spill onto the top of the right foot. Ever since, contact in this area triggered severe pain and blister formation (shown) followed by ulceration. Inability to wear a shoe precluded employment. Reproduced with permission from [31] refractory CRPS. Among 6 patients with long-standing CRPS, some benefited from therapeutic plasma exchange [69]. One open-label study in 11 patients with CRPS and 1 well-designed, RCT in 12 patients reported modest efficacy of IVIg, $1 \mathrm{~g} / \mathrm{kg}[70,71]$. Given the desperate situation of patients with refractory CRPS, some of whom resort to limb amputation to try and reduce their pain, larger trials of IVIg and other immunomodulatory therapies seem indicated.

\section{Small-fiber Polyneuropathy}

The term "polyneuropathy" refers to widespread damage throughout the peripheral nerves. Polyneuropathies usually manifest at the distal ends of the axons, which are most vulnerable to disruptions in supply of energy or nutrients. Small-fiber polyneuropathy (SFPN) typically presents with chronic widespread pain starting in the feet, and/or dysautonomic symptoms, most often cardiovascular or gastrointestinal. The cardiovascular symptoms include not only orthostatic hypotension and tachycardia, but also impaired cognitive function, headache, and exercise intolerance due to circulatory insufficiency [72-74]. Gastrointestinal symptoms can be upper or lower, and reflect dysmotility and inadequate splanchnic circulation [75, 76]. SFPN cannot be detected by electromyography or standard nerve conduction studies, so special tests are needed for objective confirmation of diagnosis. Given the nonspecificity of most SFPN symptoms, objective confirmation is routine and usually required to obtain authorization for expensive treatments. Neurological societies endorse the use of biopsies of skin biopsies from the distal leg (Fig. 2), autonomic function testing (AFT), which detects autonomic and sudomotor small-fiber activity, and surgical biopsy of sensory nerves, usually the sural [77, 78].

Skin biopsy is the most accessible test as it can be performed in any medical facility and mailed to an accredited laboratory. The distal leg is biopsied $10 \mathrm{~cm}$ above the lateral malleolus, using subcutaneous bicarbonate-buffered lidocaine. After transporting the single $3-\mathrm{mm}$ punch to the laboratory in the correct fixative, biopsies are vertically sectioned and immunolabeled against PGP9.5, a pan-neuronal marker that renders axons visible by light microscopy. As the epidermis is innervated almost exclusively by small fibers, measured innervation densities below the fifth centile of a normal distribution model are universally accepted as pathological confirmation of SFPN [77, 78]. A caveat is that each laboratory's assessment of the normality of a patient's measured density depends on the accuracy of their normative data and their statistical model. We reported that separate norms and modeling are needed to improve diagnostic accuracy in patients younger than their mid-20s [79]. Distal leg skin biopsies have high positive predictive value but lower negative predictive value. A second biopsy from the thigh is sometimes added to improve sensitivity and inform about length dependence.

Like other polyneuropathies, SFPN is caused by underlying medical problems that must be identified for optimal treatment. Diabetes and toxic causes are best known [80], but several systemic autoimmune conditions are also associated [14]. In addition to ganglionitis, Sjögren's also causes painful distal SFPN [81]. In a series of 20 pathologically confirmed SS cases, one-third had pure SFN, one-third had mixed large and small fiber involvement, and one-third were not fully characterizable [17]. My group found SS-A/SS-B antibodies in $9.2 \%$ of the 98 tested patients with objectively verified SFPN [82]. Various neuronal antigens have been implicated in Sjögren syndrome (SS) axonopathy, but verification has been elusive (reviewed in [83]). A French multicenter study documented efficacy of IVIg $2 \mathrm{~g} / \mathrm{kg}$ for SS neuropathy, including sensorimotor axonopathy [23]. 


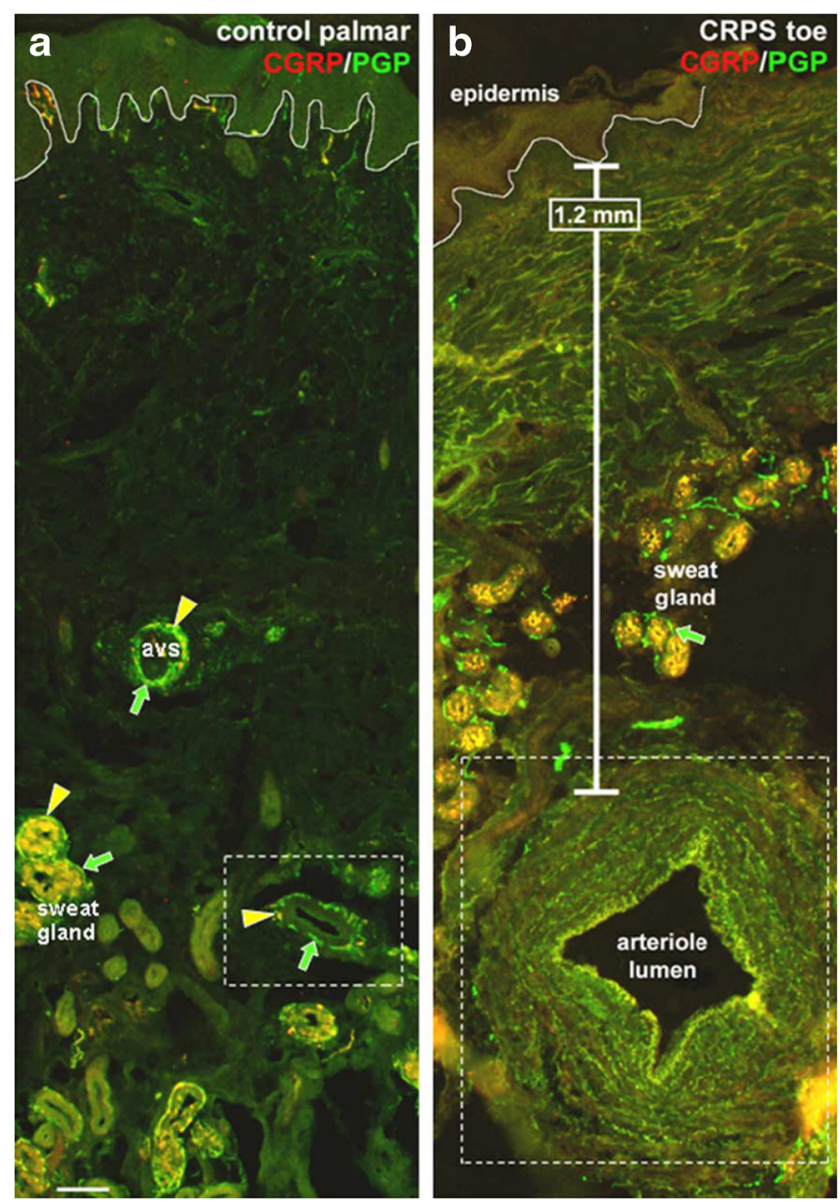

Fig. 4 Immunohistochemical labeling of vertically sectioned skin biopsies illustrates axonal origin of complex regional pain syndrome (CRPS) features. CGRP (red) and PGP9.5 (green) immunolabeling of skin axons. (A) Control palmar skin demonstrating abundant normal innervation of sweat glands, arteries (box), and arteriovenous shunts (avs) located within $2 \mathrm{~mm}$ of the epidermis. The basement membrane is delineated by a white line. All abundantly express PGP9.5, a panaxonal marker (arrows) and some co-express CGRP, a C-fiber neuropeptide (arrowheads). (B) Skin from a patient with CRPS contained sweat glands with reduced $\mathrm{PGP}^{+}$-innervation (arrow), no CGRP coexpression, and denervated, hypertrophied arterioles (box). Scale bar= $100 \mu \mathrm{m}$. Reproduced with permission from [43]

Celiac disease (sprue) is another autoimmune disease and distal SFPN, as well as ataxia and large-fiber polyneuropathy $[12,13]$. Symptoms develop when susceptible individuals carrying high-risk human leukocyte antigen alleles ingest wheat, rye, or barley. The classical symptoms are chronic malabsorptive diarrhea, flatulence, iron deficiency anemia, and weight loss, but neurological manifestations are common, even in the roughly half with "silent celiac" who lack gastrointestinal symptoms. In a series of 409 British patients with celiac disease, $13 \%$ had clinical and neurophysiological signs of sensory neuropathy [12]. A pathological survey of $>28,000$ Swedish patients with endoscopic verification of celiac identified a 2.8-times increased risk of chronic inflammatory demyelinating neuropathy, a 4.2-times increased risk of autonomic neuropathy, and a 7.6-times higher risk of mononeuritis multiplex, but no association with acute inflammatory demyelinating polyneuropathy [84]. A study of 20 patients with idiopathic SFPN found that $8 \%$ had endoscopically confirmed celiac disease [85]. My group found antiTTG-IgA antibody markers for celiac disease in $3.5 \%$ of 109 patients with "idiopathic" SFPN [82].

Symptoms of celiac disease-associated neuropathy include asymmetrical numbness or paresthesias of the limbs, hands, feet, or face, and mild-to-moderate sensory ataxia, often with a nonlength-dependent distribution [13]. Neurological manifestations appear mediated by both cellular and humoral responses, and autopsy study of celiac-affected dorsal root ganglia showed lymphocytic infiltrates with perivascular cuffing [12]. Skin biopsies show reduced epidermal innervation (Fig. 2), often in a nonlength-dependent pattern [13]. IgG antibodies against gliadin, IgG-deamidated gliadin peptide antibodies, IgA antibodies against endomysium, and IgA antibodies against different transglutaminases are also implicated (reviewed in [86]). Not all celiac neuropathy is immunological - malabsorptive vitamin deficiencies can contribute [87], although typically these present as cerebellar/ataxia rather than SFN. The cornerstone treatment, a lifelong gluten-free diet, is difficult. Immunotherapies are sometimes added for very ill patients [12], but there are no RCTs. One case series of 3 patients with biopsy-proven celiac disease reported that both their cerebellar ataxia and painful SFN improved after adding IVIg to their gluten-free diets [88].

In additional to the above systemic autoimmune causes of SFN, there is emerging evidence that some cases of SFPN of unknown cause ("idiopathic SFPN") are caused by acute or chronic tissue-specific dysimmunity, akin to the tissuespecific dysimmune large-fiber neuropathies Guillain-Barré syndrome and chronic inflammatory neuropathy. Several authors have documented steroid-responsive cases of idiopathic SFPN $[89,90]$. My patient, depicted in Fig. 5, had no history of autoimmunity or serological markers but his severe refractory neuropathic limb pain, swelling and redness responded within hours to intravenous methylprednisolone, his symptoms resolved within a year and no longer required treatment, and he remained without treatment or recurrence for more than a decade, consistent with an acute monophasic autoimmune form of SFPN [90].

This report brought referrals of other young patients, mostly with chronic courses, and we published a series of 41 patients with unexplained widespread chronic pain before the age of 21 years [74]. Objective testing revealed definite SFPN in $59 \%$ and probable SFPN in $17 \%$ of cases. Among them, $98 \%$ also had cardiovascular, gastrointestinal, or urological symptoms, documenting the unity of dysautonomic and somatic symptoms in SFPN. Exhaustive testing for causes of this "early-onset SFPN" identified only histories of autoimmune illnesses in $33 \%$ of patients and serologic 


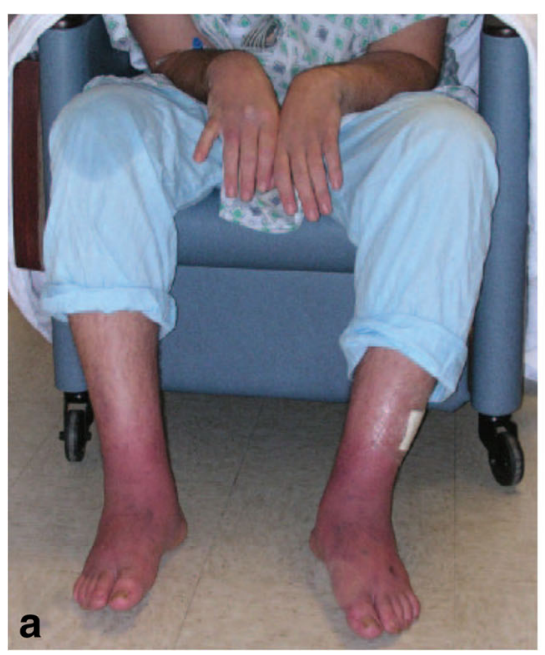

Fig. 5 A previously healthy 20 -year-old with sudden severe corticosteroid-responsive small-fiber polyneuropathy. (A) At 5 weeks postonset, just before immunotherapy, there is severe distal-limb pain, edema, redness, early tissue necrosis, and sensory loss. Dysautonomic symptoms included tachycardia and hypertension. High-dose opioids and gabapentin were ineffective for pain and he immersed his hands and feet in cold water to reduce his pain (erythromelalgia). The right

markers of disordered immunity in $89 \%$. Treatment with immunomodulatory therapy benefited $80 \%$ of 15 treated patients [74]. These reports tentatively characterize acute and chronic dysimmune forms of SFPN. The limited evidence available so far, such as normal cerebrospinal fluid, lack of cellularity within nerve biopsies, and presence of antinuclear antibodies and low serum complement $\mathrm{C} 3$ and $\mathrm{C} 4$, suggests innate and autoantibody-mediated immunity, most likely involving the classical or lectin rather than the alternative complement pathway [74].

These reports also provide a rationale for treating carefully selected patients with immunomodulatory therapies. Candidates should have symptoms consistent with SFPN, objective confirmation of diagnosis, exclusion of other causes, failure to improve with symptom management, and, ideally, serologic markers of immunity/inflammation. Given the lack of other options, and the disability of chronic widespread pain, particularly for children and young adults, it is reasonable to at least consider immunomodulatory treatments proven effective for other autoimmune neuropathies. In our series, among the 15 earlyonset patients treated with 1-2-month trials of corticosteroids $67 \%$ improved and 1 patient developed cataracts. Among the 11 early-onset patients treated at least 3 times with IVIg, $2 \mathrm{~g} / \mathrm{kg} / \mathrm{month}, 38 \%$ did not respond and discontinued treatment, and $62 \%$ had significant improvement and continued treatment [74]. Adverse events were as expected and largely manageable. Repeat skin biopsies and AFT provided objective confirmation of axonal regeneration.

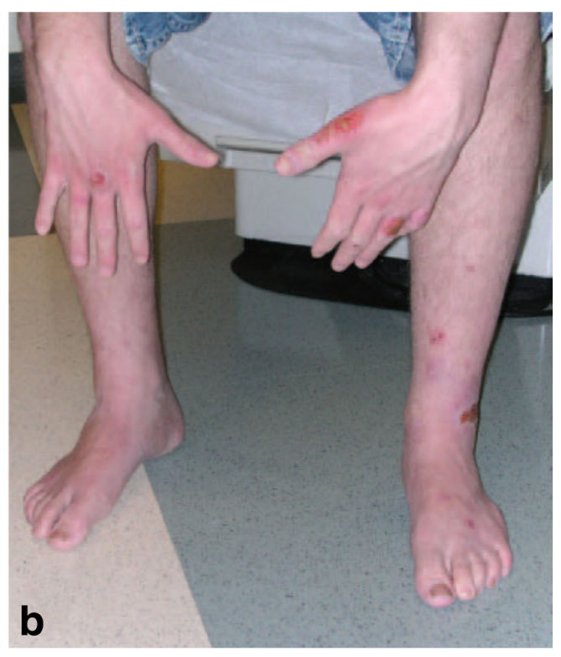

hand and left foot have early skin ulcers from tissue ischemia. (B) High-dose corticosteroids resulted in immediate improvement in all signs and symptoms, including pain. One month later during prednisone taper, the redness and edema were resolved, the ulcers were healing, and the left first dorsal interosseous muscle had neurogenic atrophy, consistent with bystander damage to myelinated motor fibers. Reproduced with permission from [90]

We recently reviewed the outcomes of 46 consecutive patients of all ages with objectively confirmed SFPN, dysimmune attribution, and treatment with $\geq 1$ cycle of IVIg at $2 \mathrm{~g} / \mathrm{kg}$ for 4 weeks; the primary outcome was change in pain [91]. Among the 31 with baseline pain scores $\geq 3$, pretreatment pain was significantly reduced, and among the 28 patients with pre- and post-treatment AFT, the high prevalence of abnormal AFT at baseline was halved. Patients' global impression-of-change scores showed that $>70 \%$ rated themselves as improved [91]. Thus, the preliminary evidence supports the use of shortterm corticosteroids and long-term IVIg for carefully selected patients with SFPN. These retrospective data also provide a rationale for conducting prospective studies and for defining the mechanisms of autoimmune SFPN.

A new discovery is that roughly half of patients with fibromyalgia have objective evidence of SFPN. The label "fibromyalgia" describes a symptom complex that previously lacked any link to pathobiology, leaving practitioners attributing it to psychopathology or unspecified "central sensitization". As fibromyalgia affects several percent of the population, the public health and economic implications are considerable. Initial pathology skin biopsy studies identifying loss of cutaneous innervation were followed by electrophysiologic evidence that C-fibers fire spontaneously and excessively in patients with fibromyalgia as in positive-control patients with SFPN but not normal controls [74, 92-94]. Subsequent studies have so far all been supportive (e.g., [95]), so biological overlap between fibromyalgia and 
SFPN appears confirmed. "Fibromyalgia" is a label applied to a family of multisymptom disorders with different underlying causes, and it may be superseded, as the old term "pulmonary congestion" was replaced by specific pathological diagnoses, for example heart failure, tuberculosis, and lung cancer.

This new discovery is not yet widely known but requests to evaluate patients with fibromyalgia for SFPN are already rising. They seem justified as SFPN has identifiable underlying causes that can sometimes be definitively treated and occasionally cured. However, neither skin biopsy nor microneurography are widely available, so simpler diagnostic tools are needed. The implications for immunotherapy are net yet certain. A proportion of patients with fibromyalgia have evidence for inflammation/dysimmune mechanisms by virtue of also having systemic autoimmune diseases, dysimmune profiles in blood or skin, and/or improvement in fibromyalgia symptoms after immunotherapy [93]. Comparing patients with fibromyalgia with those without systemic autoimmune rheumatologic disorders revealed that $44 \%$ of those with systemic autoimmunity versus $27 \%$ of those without it had skin biopsies consistent with SFPN [95]. Thus, it may be reasonable to more seriously consider immunotherapy for the subset of patients with fibromyalgia with confirmed SFPN, evidence of immune mediation, exclusion of other causes, and inadequate response to symptom-based treatments.

\section{Conclusion}

The development of objective methods of detecting small-fiber loss, most notably skin biopsies immunolabeled to reveal small-fiber innervation (Fig. 2), is beginning to improve awareness and detection of small-fiber illnesses. They are more common and diverse than appreciated. There is now preliminary evidence of dysimmune causes of some neuropathies that present primarily with neuropathic pain or dysautonomic symptoms, particularly in patients with Sjögren, celiac disease, or paraneoplastic syndromes. Limited data now suggest that dysimmune SFNs respond to immunotherapies like other autoimmune neurological conditions. Given the distress, disability, high cost, and prevalence of chronic pain and widespread multisymptom illnesses, the need to establish and validate outcome measures, verify the proportion of illnesses caused by dysimmunity, dissect molecular mechanisms, and conduct prospective trials of immunotherapies is becoming evident.

Acknowledgments This work was supported, in part, by the National Institutes of Health (K24NS059892, R01NS093653). I acknowledge the mentorship of the late John W. Griffin MD with gratitude.

\section{Compliance with ethical standards}

Required Author Forms Disclosure forms provided by the authors are available with the online version of this article.

\section{References}

1. Fukuda $\mathrm{T}$, Takeda $\mathrm{S}, \mathrm{Xu} \mathrm{R}$, et al. Sema3A regulates bone-mass accrual through sensory innervations. Nature 2013 23;497:490493.

2. Hsieh ST, Lin WM. Modulation of keratinocyte proliferation by skin innervation. J Invest Dermatol 1999;113:579-586.

3. Levine J, Dardick SJ, Basbaum AI, Scipio E. Reflex neurogenic inflammation 1. contribution of the peripheral nervous system to spatially remote inflammatory responses that follow injury. J Neurosci 1985;5:1380-1386.

4. Egan CL, Viglione-Schneck MJ, Walsh LJ, et al. Characterization of unmyelinated axons uniting epidermal and dermal immune cells in primate and murine skin. J Cutan Pathol 1998;25:20-29.

5. Basbaum AI, Bautista DM, Scherrer G, Julius D. Cellular and molecular mechanisms of pain. Cell 2009 16;139:267-284

6. McMahon SB, Russa FL, Bennett DL. Crosstalk between the nociceptive and immune systems in host defence and disease. Nat Rev Neurosci 2015 19;16:389-402.

7. Kuntzer T, Antoine JC, Steck AJ. Clinical features and pathophysiological basis of sensory neuronopathies (ganglionopathies). Muscle Nerve 2004;30:255-268.

8. Sheikh SI, Amato AA. The dorsal root ganglion under attack: the acquired sensory ganglionopathies. Pract Neurol 2010;10:326-334.

9. Cole JD, Katifi HA. Evoked potentials in a man with a complete large myelinated fibre sensory neuropathy below the neck. Electroencephalogr Clin Neurophysiol 1991;80:103-107.

10. Griffin JW, Cornblath DR, Alexander E, et al. Ataxic sensory neuropathy and dorsal root ganglionitis associated with Sjögren's syndrome. Ann Neurol 1990;27:304-315.

11. Lauria G, Sghirlanzoni A, Lombardi R, Pareyson D. Epidermal nerve fiber density in sensory ganglionopathies: clinical and neurophysiologic correlations. Muscle Nerve 2001;24:1034-1039.

12. Hadjivassiliou M, Rao DG, Wharton SB, Sanders DS, Grunewald RA, Davies-Jones AGB. Sensory ganglionopathy due to gluten sensitivity. Neurology 2010 14;75:1003-1008.

13. Brannagan TH, III, Hays AP, Chin SS, et al. Small-fiber neuropathy/neuronopathy associated with celiac disease: skin biopsy findings. Arch Neurol 2005 1;62:1574-1578.

14. Sene D, Cacoub P, Authier FJ, et al. Sjögren syndrome-associated small fiber neuropathy: Characterization from a prospective series of 40 cases. Medicine 2013 Aug 26 [Epub ahead of print].

15. Sene D, Jallouli M, Lefaucheur JP, et al. Peripheral neuropathies associated with primary Sjogren syndrome: immunologic profiles of nonataxic sensory neuropathy and sensorimotor neuropathy. Medicine 2011;90:133-138.

16. Birnbaum J. Peripheral nervous system manifestations of Sjögren syndrome: clinical patterns, diagnostic paradigms, etiopathogenesis, and therapeutic strategies. Neurologist 2010;16: 287-297.

17. Chai J, Herrmann DN, Stanton M, Barbano RL, Logigian EL. Painful small-fiber neuropathy in Sjögren's syndrome. Neurology 2005 27;65:925-927.

18. Brito-Zeron P, Ramos-Casals M. Advances in the understanding and treatment of systemic complications in Sjogren's syndrome. Curr Opin Rheumatol 2014;26:520-527. 
19. Gheitasi H, Kostov B, Solans R, et al. How are we treating our systemic patients with primary Sjogren syndrome? Analysis of 1120 patients. Int Immunopharmacol 2015;27:194-199.

20. Gottenberg JE, Ravaud P, Puechal X, et al. Effects of hydroxychloroquine on symptomatic improvement in primary Sjogren syndrome: the JOQUER randomized clinical trial. JAMA 2014;312:249-258.

21. Devauchelle-Pensec V, Mariette X, Jousse-Joulin S, et al. Treatment of primary Sjogren syndrome with rituximab: a randomized trial. Ann Intern Med 2014;160:233-242.

22. Takahashi Y, Takata T, Hoshino M, Sakurai M, Kanazawa I. Benefit of IVIG for long-standing ataxic sensory neuronopathy with Sjögren's syndrome. IV immunoglobulin. Neurology 2003;60: 503-505.

23. Rist S, Sellam J, Hachulla E, et al. Experience of intravenous immunoglobulin therapy in neuropathy associated with primary Sjögren's syndrome: a national multicentric retrospective study. Arthritis Care Res 2011;63:1339-1344.

24. Graus F, Keime-Guibert F, Rene R, et al. Anti-Hu-associated paraneoplastic encephalomyelitis: analysis of 200 patients. Brain 2001;124:1138-1148.

25. Kalanie H, Harandi AA, Mardani M, et al. Trigeminal neuralgia as the first clinical manifestation of anti-hu paraneoplastic syndrome induced by a borderline ovarian mucinous tumor. Case Rep Neurol 2014;6:7-13.

26. Vedeler CA, Antoine JC, Giometto B, et al. Management of paraneoplastic neurological syndromes: report of an EFNS Task Force. Eur J Neurol 2006;13:682-690.

27. Honnorat J. Therapeutic approaches in antibody-associated central nervous system pathologies. Rev Neurol (Paris) 2014;170:587594.

28. Honnorat J. Early-onset immunotherapy by intravenous immunoglobulin and corticosteroids in well characterized onconeuralantibody-positive paraneoplastic neurological syndrome. Clin Exp Immunol 2014;178(Suppl. 1):127-129.

29. Coret F, Bosca I, Fratalia L, Perez-Griera J, Pascual A, Casanova B. Long-lasting remission after rituximab treatment in a case of anti$\mathrm{Hu}$-associated sensory neuronopathy and gastric pseudoobstruction. J Neurooncol 2009;93:421-423.

30. Oaklander AL. The density of remaining nerve endings in human skin with and without postherpetic neuralgia after shingles. Pain 2001;92:139-145.

31. Oaklander AL, Fields HL. Is reflex sympathetic dystrophy/complex regional pain syndrome type I a small-fiber neuropathy? Ann Neurol 2009;65:629-638.

32. de Rooij AM, Perez RSGM, Huygen FJ, et al. Spontaneous onset of complex regional pain syndrome. Eur J Pain 2010;14:510-513.

33. Förster M, Umnus A, Siebrecht D, Baron R, Wasner G. A case of pain, motor impairment, and swelling of the arm after acute herpes zoster infection. Pain 2012;153:2478-2481.

34. Merskey H, Bogduk N. Classification of chronic pain: descriptions of chronic pain syndromes and definitions of pain terms. 2nd ed. IASP Press, Seattle, 1994.

35. Mitchell SW. Injuries of nerves and their consequences. Dover Publications, American Academy of Neurology Reprint Series (1965 edition), New York, 1864.

36. Mitchell SW, Morehouse GR, Keen WM. Gunshot wounds and other injuries of nerves. J.B. Lippincott, Philadelphia, PA, 1864.

37. Sudeck PHM. Über die akute (reflektorische) Knochenatrophie nach Entzündungen und Verletzungen an den Extremitäten und ihre klinischen Erscheinungen. Fortschritte auf dem Gebiete der Röntgenstrahlen 1901;5:277.

38. Kozin F, McCarty DJ, Sims J, Genant H. The reflex sympathetic dystrophy syndrome. I. Clinical and histologic studies: evidence for bilaterality, response to corticosteroids and articular involvement. Am J Med 1976;60:321-331.
39. Kozin F, Genant HK, Bekerman C, McCarty DJ. The reflex sympathetic dystrophy syndrome. II. Roentgenographic and scintigraphic evidence of bilaterality and of periarticular accentuation. Am J Med 1976;60:332-338.

40. Evans JA. Reflex sympathetic dystrophy. Surg Gynecol Obstet 1946;82:36-44.

41. Horowitz SH. Peripheral nerve injury and causalgia secondary to routine venipuncture. Neurology 1994;44:962-964.

42. Oaklander AL, Rissmiller JG, Gelman LB, Zheng L, Chang Y, Gott R. Evidence of focal small-fiber axonal degeneration in complex regional pain syndrome-I (reflex sympathetic dystrophy). Pain 2006;120:235-243.

43. Albrecht PJ, Hines S, Eisenberg E, et al. Pathologic alterations of cutaneous innervation and vasculature in affected limbs from patients with complex regional pain syndrome. Pain 2006;120:244-266.

44. van der Laan L, ter Laak HJ, Gabreels-Festen A, Gabreels F, Goris RJ. Complex regional pain syndrome type I (RSD): pathology of skeletal muscle and peripheral nerve. Neurology 1998;51:20-25.

45. de Mos M, Huygen FJ, Dieleman JP, Koopman JS, Stricker BH, Sturkenboom MC. Medical history and the onset of complex regional pain syndrome (CRPS). Pain 2008;139:458-466.

46. Beerthuizen A, Spijker A, Huygen FJPM, Klein J, de Wit R. Is there an association between psychological factors and the Complex Regional Pain Syndrome type 1 (CRPS1) in adults? A systematic review. Pain 2009;145:52-59.

47. Sandroni P, Benrud-Larson LM, McClelland RL, Low PA. Complex regional pain syndrome type I: incidence and prevalence in Olmsted county, a population-based study. Pain 2003;103:199 207.

48. Huge V, Lauchart M, Magerl W, et al. Complex interaction of sensory and motor signs and symptoms in chronic CRPS. PLoS ONE 2011;6:e18775.

49. Birklein F, Riedl B, Sieweke N, Weber M, Neundörfer B. Neurological findings in complex regional pain syndromesanalysis of 145 cases. Acta Neurol Scand 2000;101:262-269.

50. Tilman PBJ, Stadhouders AM, Jap PHK, Goris RJA. Histopathologic findings in skeletal muscle tissue of patients suffering from reflex sympathetic dystrophy. Micron Microscop Acta 1990;21:271-272.

51. Weber M, Birklein F, Neundörfer B, Schmelz M. Facilitated neurogenic inflammation in complex regional pain syndrome. Pain 2001;91:251-257.

52. Webster GF, Iozzo RV, Schwartzman RJ, Tahmoush AJ, Knobler RL, Jacoby RA. Reflex sympathetic dystrophy: Occurrence of chronic edema and nonimmune bullous skin lesions. J Am Acad Dermatol 1993;28:29-32.

53. Eltzschig HK, Carmeliet P. Hypoxia and inflammation. N Engl J Med 2011;364:656-665.

54. Birklein F, Weber M, Ernst M, Riedl B, Neundörfer B, Handwerker HO. Experimental tissue acidosis leads to increased pain in complex regional pain syndrome (CRPS). Pain 2001;87:227-234.

55. Parkitny L, McAuley JH, Di Pietro F, et al. Inflammation in complex regional pain syndrome: A systematic review and meta-analysis. Neurology 2013 1;80:106-117.

56. Lenz M, Üceyler N, Frettloh J, et al. Local cytokine changes in complex regional pain syndrome type I (CRPS I) resolve after 6 months. Pain 2013;154:2142-2149.

57. Iwatsuki $\mathrm{K}$, Arai $\mathrm{T}$, Ota $\mathrm{H}$, et al. Targeting anti-inflammatory treatment can ameliorate injury-induced neuropathic pain. PLoS One 2013;8:e57721.

58. Üçeyler N, Rogausch JP, Toyka KV, Sommer C. Differential expression of cytokines in painful and painless neuropathies. Neurology 2007 3;69:42-49.

59. Kingery WS. A critical review of controlled clinical trials for peripheral neuropathic pain and complex regional pain syndromes. Pain 1997;73:123-139. 
60. Eisenberg E, Sandler I, Treister R, Suzan E, Haddad M. Anti tumor necrosis factor - alpha adalimumab for complex regional pain syndrome type 1 (CRPS-I): A case series. Pain Pract 2013;13:649-656.

61. Schwartzman RJ, Chevlen E, Bengtson K. Thalidomide has activity in treating complex regional pain syndrome. Arch Intern Med 2003 23;163:1487-1488.

62. Manning DC, Alexander G, Arezzo JC, et al. Lenalidomide for complex regional pain syndrome type 1: Lack of efficacy in a phase II randomized study. J Pain 2014;15:1366-1376.

63. Goebel A, Blaes F. Complex regional pain syndrome, prototype of a novel kind of autoimmune disease. Autoimmun Rev 2013;12:682686.

64. Kohr D, Tschernatsch M, Schmitz K, et al. Autoantibodies in complex regional pain syndrome bind to a differentiation-dependent neuronal surface autoantigen. Pain 2009;143:246-251.

65. Kohr D, Singh P, Tschernatsch M, et al. Autoimmunity against the beta(2) adrenergic receptor and muscarinic-2 receptor in complex regional pain syndrome. Pain 2011;152:2690-2700.

66. Tekus V, Hajna Z, Borbely E, et al. A CRPS-IgG-transfer-trauma model reproducing inflammatory and positive sensory signs associated with complex regional pain syndrome. Pain 2014;155:299-308.

67. Li WW, Guo TZ, Shi X, et al. Autoimmunity contributes to nociceptive sensitization in a mouse model of complex regional pain syndrome. Pain 2014;155:2377-2389.

68. Staff NP, Engelstad J, Klein CJ, et al. Post-surgical inflammatory neuropathy. Brain 2010;133:2866-2880.

69. Goebel A, Jones S, Oomman S, Callaghan T, Sprotte G. Treatment of long-standing complex regional pain syndrome with therapeutic plasma exchange: a preliminary case series of patients treated in 2008-2014. Pain Med 2014;15:2163-2164.

70. Goebel A, Netal S, Schedel R, Sprotte G. Human pooled immunoglobulin in the treatment of chronic pain syndromes. Pain Med 2002;3:119-127.

71. Goebel A, Baranowski A, Maurer K, Ghiai A, McCabe C, Ambler G. Intravenous immunoglobulin treatment of the complex regional pain syndrome: a randomized trial. Ann Intern Med 2010 2;152: 152-158.

72. Benarroch EE. Postural tachycardia syndrome: a heterogeneous and multifactorial disorder. Mayo Clin Proc 2012;87:1214-1225.

73. Poda R, Guaraldi P, Solieri L, et al. Standing worsens cognitive functions in patients with neurogenic orthostatic hypotension. Neurol Sci 2012;33:469-473.

74. Oaklander AL, Klein MM. Evidence of small-fiber polyneuropathy in unexplained, juvenile-onset, widespread pain syndromes. Pediatrics 2013;131:e1091-e1100.

75. Parkman HP, Yates K, Hasler WL, et al. Clinical features of idiopathic gastroparesis vary with sex, body mass, symptom onset, delay in gastric emptying, and gastroparesis severity. Gastroenterology 2011;140:101-115.

76. Pasricha PJ, Colvin R, Yates K, et al. Characteristics of patients with chronic unexplained nausea and vomiting and normal gastric emptying. Clin Gastroenterol Hepatol 2011;9:567-576.

77. England JD, Gronseth GS, Franklin G, et al. Practice Parameter: Evaluation of distal symmetric polyneuropathy: role of autonomic testing, nerve biopsy, and skin biopsy (an evidence-based review). Report of the American Academy of Neurology, American Association of Neuromuscular and Electrodiagnostic Medicine, and American Academy of Physical Medicine and Rehabilitation. Neurology 2009 13;72:177-184.
78. Lauria G, Hsieh ST, Johansson O, et al. European Federation of Neurological Societies/Peripheral Nerve Society Guideline on the use of skin biopsy in the diagnosis of small fiber neuropathy. Report of a joint task force of the European Federation of Neurological Societies and the Peripheral Nerve Society. Eur J Neurol 2010;17: 903-909.

79. Klein MM, Downs H, O'Neil K., Oaklander AL. Skin biopsy of normal children demonstrates inverse correlation between age and epidermal nerve fiber density, meaning age-specific norms are needed. Ann Neurology 2014;76:S69

80. Bednarik J, Vlckova-Moravcova E, Bursova S, Belobradkova J, Dusek L, Sommer C. Etiology of small-fiber neuropathy. J Peripher Nerv Syst 2009;14:177-183.

81. Gorson KC, Ropper AH. Positive salivary gland biopsy, Sjögren's syndrome, and neuropathy: clinical implications. Muscle Nerve 2003;28:553-560.

82. Lang M, Treister R, Oaklander AL. Cost/benefit of blood tests in idiopathic small-fiber polyneuropathy (SFPN). Ann Neurol 2015;78:S19.

83. Pavlakis PP, Alexopoulos H, Kosmidis ML, et al. Peripheral neuropathies in Sjögren's syndrome: a critical update on clinical features and pathogenetic mechanisms. J Autoimmun 2012;39:27-33.

84. Thawani SP, Brannagan TH, III, Lebwohl B, Green PH, Ludvigsson JF. Risk of neuropathy among 28232 patients with biopsy-verified celiac disease. JAMA Neurol 2015;72:806-811.

85. Chin RL, Sander HW, Brannagan TH, et al. Celiac neuropathy. Neurology 2003 27;60:1581-1585.

86. Martinez AR, Nunes MB, Nucci A, Franca MC, Jr. Sensory neuronopathy and autoimmune diseases. Autoimmune Dis 2012;2012:873587.

87. McKeon A, Lennon VA, Pittock SJ, Kryzer TJ, Murray J. The neurologic significance of celiac disease biomarkers. Neurology 2014;83:1789-1796.

88. Souayah N, Chin RL, Brannagan TH, et al. Effect of intravenous immunoglobulin on cerebellar ataxia and neuropathic pain associated with celiac disease. Eur J Neurol 2008;15:1300-1303.

89. Dabby R, Gilad R, Sadeh M, Lampl Y, Watemberg N. Acute steroid responsive small-fiber sensory neuropathy: a new entity? J Peripher Nerv Syst 2006;11:47-52.

90. Paticoff J, Valovska A, Nedeljkovic SS, Oaklander AL. Defining a treatable cause of erythromelalgia: acute adolescent autoimmune small-fiber axonopathy. Anesth Analg 2007;104:438-441.

91. Oaklander AL, Lang M, Treister R, Liu X. First evaluation of immunoglobulin treatment of small-fiber axonal polyneuropathy (SFPN). Ann Neurol 2015;78:S109.

92. Oaklander AL, Herzog ZD, Downs HM, Klein MM. Objective evidence that small-fiber polyneuropathy underlies some illnesses currently labeled as fibromyalgia. Pain 2013;154:2310-2316.

93. Üçeyler N, Zeller D, Kahn AK, et al. Small fibre pathology in patients with fibromyalgia syndrome. Brain 2013;136:1857-1867.

94. Serra J, Collado A, Sola R, et al. Hyperexcitable C nociceptors in fibromyalgia. Ann Neurol 2013;75:196-208.

95. Kosmidis ML, Koutsogeorgopoulou L, Alexopoulos H, et al. Reduction of intraepidermal nerve fiber density (IENFD) in the skin biopsies of patients with fibromyalgia: A controlled study. J Neurol Sci 2014;347:143-147.

96. Harden RN, Bruehl S, Perez RSGM, et al. Validation of proposed diagnostic criteria (the Budapest Criteria) for Complex Regional Pain Syndrome. Pain 2010;150:268-274. 